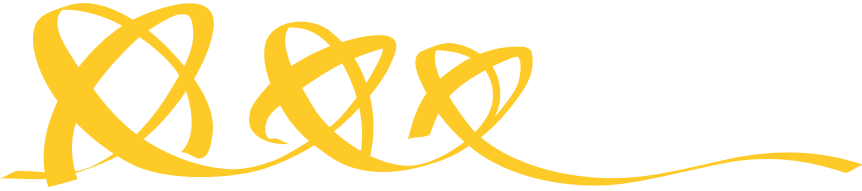 COMMUNICATIONS PHYSICS
}

PERSPECTIVE

\section{Probing light-driven quantum materials with ultrafast resonant inelastic X-ray scattering}

\author{
Matteo Mitrano ${ }^{1 凶} \&$ Yao Wang (iD ${ }^{2 凶}$
}

Ultrafast optical pulses are an increasingly important tool for controlling quantum materials and triggering novel photo-induced phase transitions. Understanding these dynamic phenomena requires a probe sensitive to spin, charge, and orbital degrees of freedom. Timeresolved resonant inelastic X-ray scattering (trRIXS) is an emerging spectroscopic method, which responds to this need by providing unprecedented access to the finite-momentum fluctuation spectrum of photoexcited solids. In this Perspective, we briefly review state-ofthe-art trRIXS experiments on condensed matter systems, as well as recent theoretical advances. We then describe future research opportunities in the context of light control of quantum matter.

nderstanding and controlling quantum materials - material systems exhibiting quantummechanical effects over wide energy and length scales ${ }^{1}$ - is a central challenge in modern condensed matter physics. Over the last two decades, ultrafast lasers have had a tremendous impact on quantum materials research and provided a novel route to on-demand engineering of their electronic and structural properties. They have not only allowed for tuning well-known states of matter far from equilibrium, e.g., magnetism ${ }^{2-6}$, charge/spin $\operatorname{order}^{7-11}$, and ferroelectricity ${ }^{12-14}$, but also led to novel dynamical phenomena, such as transient superconductivity ${ }^{15-18}$ and Floquet topological phases ${ }^{19-21}$.

In nonequilibrium experiments, a sample is typically excited by a pump pulse and monitored by a subsequent probe. Interpreting the properties of a photoexcited material, especially when different instabilities are intertwined, requires precise knowledge of how the lattice, band structure, and collective fluctuations respond to the pump. To meet these needs, the ultrafast community developed ultrafast X-ray and electron diffraction for monitoring the crystal lattice $^{22-25}$ and time- and angle-resolved photoemission (trARPES) for probing the electronic structure ${ }^{7,9,26}$. On the other hand, light-driven collective excitations are commonly investigated with ultrafast optical methods ${ }^{27-29}$, which however cannot probe their dispersion in reciprocal space owing to the negligible momentum of optical photons. This implies that the microscopic distribution of nonequilibrium fluctuations in quantum materials is largely inaccessible to most existing experimental methods.

\footnotetext{
${ }^{1}$ Department of Physics, Harvard University, Cambridge, MA 02138, USA. ${ }^{2}$ Department of Physics and Astronomy, Clemson University, Clemson, SC 29631

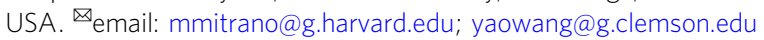


Time-resolved resonant inelastic X-ray scattering (trRIXS) is a momentum-resolved spectroscopy aimed to interrogate nonequilibrium collective modes, which has been recently enabled by the development of femtosecond X-ray free-electron lasers (XFELs) ${ }^{30,31}$. As shown in Fig. 1a, trRIXS probes nonequilibrium dynamics by scattering ultrashort X-ray pulses tuned to a characteristic atomic absorption edge. Once the incoming $\mathrm{X}$-ray photon is absorbed, the pump-excited material transitions to an intermediate state in which a core-level electron is transferred to (or above) the valence orbitals. Within a few fs, the highly unstable intermediate state decays and a valence electron fills the core hole by emitting a X-ray photon (see Fig. 1b). The scattered X-rays are then analyzed in both momentum and energy, yielding information about the pump-induced collective dynamics. The resonance condition greatly enhances the RIXS cross-section, but the many-body interactions in the intermediate state are what really makes trRIXS sensitive to a wide variety of charge, orbital, and spin excitations of the valence electrons ${ }^{32,33}$.

In this perspective, we survey recent experimental and theoretical progress in trRIXS. Then, we outline future research opportunities emerging from these new spectroscopic capabilities. While trRIXS will have a tremendous cross-disciplinary impact, ranging from chemistry ${ }^{34-36}$ to condensed matter physics, here we specifically focus on quantum materials' research.

\section{A unique experimental tool}

The Linac Coherent Light Source (LCLS) at the SLAC National Accelerator Laboratory has been at the forefront in developing trRIXS capabilities in both the soft and hard X-ray regime ${ }^{30,31}$. Over the last five years, these developments motivated a variety of experiments focused on probing nonequilibrium correlations in light-driven quantum materials, especially in connection to the problem of high- $T_{c}$ superconductivity.

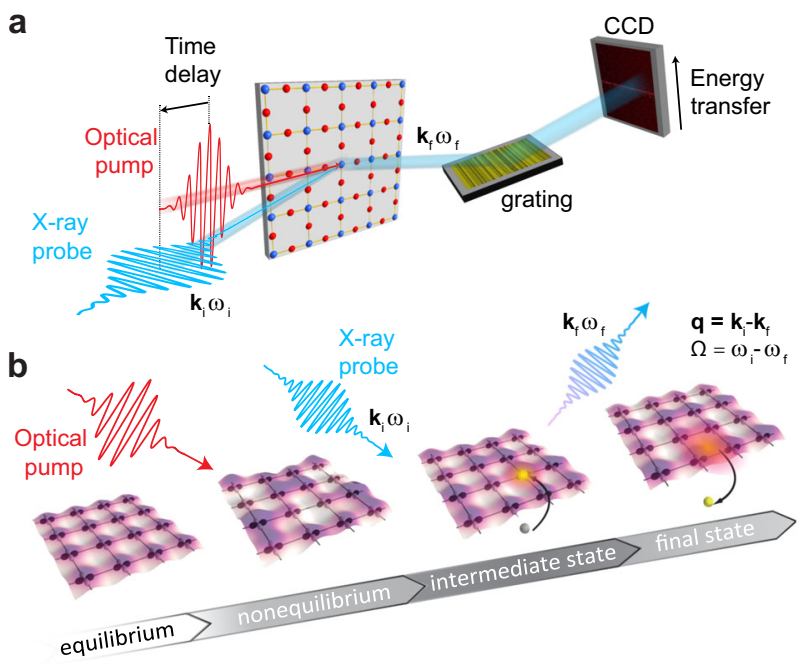

Fig. 1 Basics of a time-resolved resonant inelastic $X$-ray scattering (trRIXS) experiment. a A material is driven out of equilibrium by an ultrafast optical pump and then probed with short, delayed $X$-ray pulses with energy and momentum $\left(\mathbf{k}_{\mathbf{i}}, \omega_{i}\right)$ resonantly tuned to a specific absorption edge, which are scattered off the sample with energy and momentum $\left(\mathbf{k}_{\mathbf{f}}, \omega_{f}\right)$. The scattered photon energy is resolved by spatially separating different spectral components onto a charge-coupled device (CCD) detector with either a diffraction grating or a crystal analyzer. b Time-dependent sequence of the trRIXS process. Adapted with permission from ref. ${ }^{72}$ Copyrighted by the American Physical Society.
Key challenges in the physics of high- $T_{\mathrm{c}}$ superconductors are understanding the relationship between superconductivity and other low-temperature instabilities, as well as devising routes to further enhance $T_{c}$. In the case of copper oxides, while superconductivity appears upon doping, hole-like carriers also form unidirectional charge (and sometimes spin) order (CO) modulations close to $1 / 8$ doping and at temperatures above the superconducting $T_{c}$, which result in diffraction peaks at a finitemomentum $\mathbf{Q}_{\mathrm{CO}}{ }^{37-43}$.

Experimental and theoretical evidence suggest indeed that these two phases interplay and often compete ${ }^{39,44,45}$. Furthermore, ultrafast optical pulses have been found to enhance superconductivity while melting charge order correlations ${ }^{15-17,46-48}$, and vice versa ${ }^{49}$. Understanding how light affects the balance between these two phases and their collective dynamics with the aim of further optimizing superconductivity requires measuring the transient inelastic charge spectrum.

To this end, a recent trRIXS experiment at the $\mathrm{Cu} L$-edge investigated the light-induced charge order dynamics of the prototypical stripe-ordered cuprate $\mathrm{La}_{2-x} \mathrm{Ba}_{x} \mathrm{CuO}_{4}$ (see Fig. 2a) ${ }^{50,51}$. trRIXS spectra clearly show that $1.55-\mathrm{eV}$ photons, which transiently enhance interlayer superconducting tunneling ${ }^{46,52}$, also deplete the quasielastic charge order peak at $\mathbf{Q}_{\mathrm{CO}}$ (see Fig. 2b, c). Unlike conventional charge density waves ${ }^{53}$, the CO is found to undergo a sudden light-induced sliding motion ${ }^{51}$ and exhibit an exponential recovery dominated by yet unobserved diffusive fluctuations at the sub-meV scale ${ }^{50}$. By applying a similar approach to other copper oxides such as $\mathrm{YBa}_{2} \mathrm{Cu}_{3} \mathrm{O}_{7-\delta}$ and $\mathrm{Nd}_{1+x} \mathrm{Ba}_{2-x} \mathrm{Cu}_{3} \mathrm{O}_{7-\delta}$, we expect trRIXS to provide new insights about the broader dynamical relationship between charge order and superconductivity.

Aside from charge dynamics, spin fluctuations - particularly, near the antiferromagnetic wavevector $\mathbf{Q}_{\mathrm{AF}}=(0.5,0.5)$ reciprocal lattice units (r.l.u.) - are believed to contribute to the superconducting pairing ${ }^{55-60}$. Thus, their optical excitation is a promising route to manipulate nonequilibrium superconductivity. Being sensitive to spin degrees of freedom through the intermediate state, trRIXS is the only available method for measuring the transient magnetic excitation spectrum as a function of momentum. Pioneering experiments provided a first glimpse of light-induced spin dynamics in a Mott insulator ${ }^{61,62}$. Unlike $\mathrm{Cu} L$-edge X-rays (see Fig. 2a), photons at the $L$-edge of $5 d$ transition metals carry enough momentum to fully map magnetic fluctuations throughout the Brillouin Zone (see Fig. 3a). Among the $5 d$ materials, iridates $\left(\mathrm{Sr}_{n+1} \mathrm{Ir}_{n} \mathrm{O}_{3 n+1}\right)$ are particularly interesting analogs of copper oxides. These compounds may give rise to unconventional superconductivity upon doping ${ }^{63,64}$, with pseudospin fluctuations (owing to spin-orbit coupling) playing the same role as spins in cuprates. Driving onsite orbital excitations with infrared pump pulses resulted in a significant spectral weight reshaping of pseudospin excitations at the high-symmetry points $\mathbf{Q}_{1}=(0.5,0)$ and $\mathbf{Q}_{2}=(0.5,0.5)$ r.l.u. (see Fig. 3b, c) ${ }^{61,62}$. These first snapshots of light-stimulated magnetic excitations at the Ir $L$-edge show that optical pump pulses with a near-zero momentum transfer have profound influence on the finitemomentum spin dynamics throughout the Brillouin Zone of quantum materials.

These experiments, along with further studies of orbital excitations ${ }^{65,66}$, demonstrate how trRIXS provides simultaneous access to charge, spin, and orbital degrees of freedom far from equilibrium. Its sensitivity to collective fluctuations at large momenta fills a long-standing gap in time-resolved experiments and makes trRIXS a unique tool to advance our microscopic understanding of light-driven phenomena in quantum materials. 
a

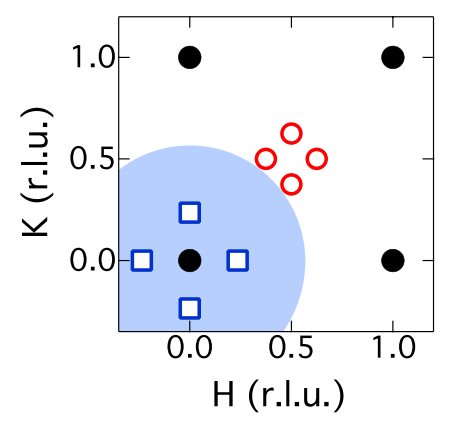

Bragg peaks

$\square$ Charge Order (CO)

O Spin Order (SO) b

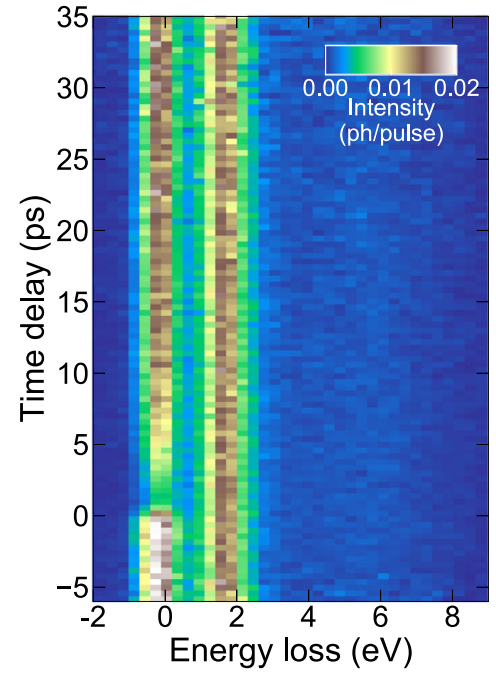

c

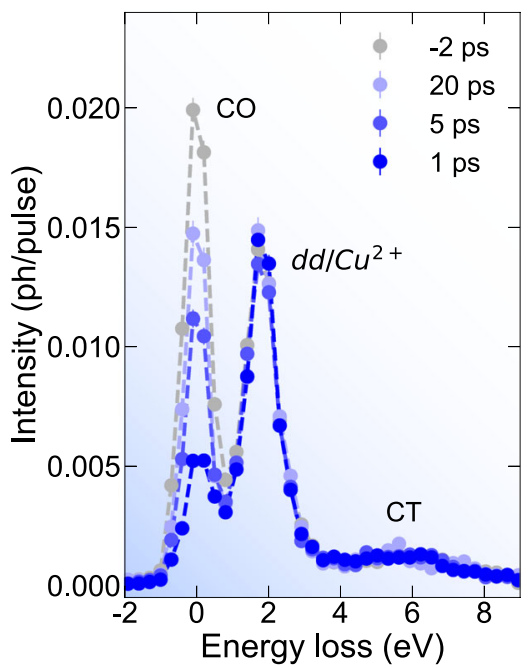

Fig. $\mathbf{2}$ Charge order melting in $\mathbf{L a}_{\mathbf{2}} \mathbf{B a}_{\mathbf{x}} \mathbf{C u O}_{\mathbf{4}}$. a Reciprocal space map of structural Bragg peaks, charge order (CO) and spin order (SO) peaks in $\mathrm{La}_{2-x} \mathrm{Ba}_{x} \mathrm{CuO}_{4}$. The blue area represents the maximum momentum transfer achievable at the $\mathrm{Cu} L$-edge (931 eV). The location of peaks in momentum space is denoted by the Miller indices $(H, K, L)$ and expressed in reciprocal lattice units (r.l.u.) b Time-resolved resonant inelastic X-ray scattering (trRIXS) spectra of photoexcited $\mathrm{La}_{1.875} \mathrm{Ba}_{0.125} \mathrm{CuO}_{4}$ at $Q_{\mathrm{CO}}=(0.236,0,1.5)$ r.l.u. at the $\mathrm{Cu}$ L-edge for variable pump-probe time delay. c trRIXS spectra for a selection of time delays showing a prompt melting of the $\mathrm{CO}$, whereas higher energy features of the inelastic spectrum, e.g., dd excitations/Cu2+ emission and charge transfer (CT) excitations, remain unaffected. Error bars represent Poisson counting uncertainties. Adapted with permission from ref. 50.

a

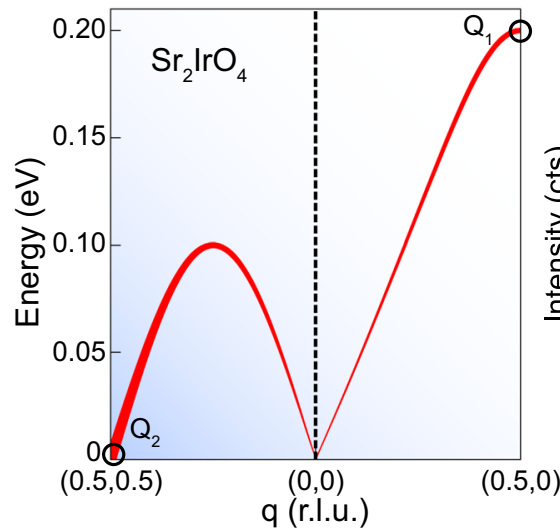

b

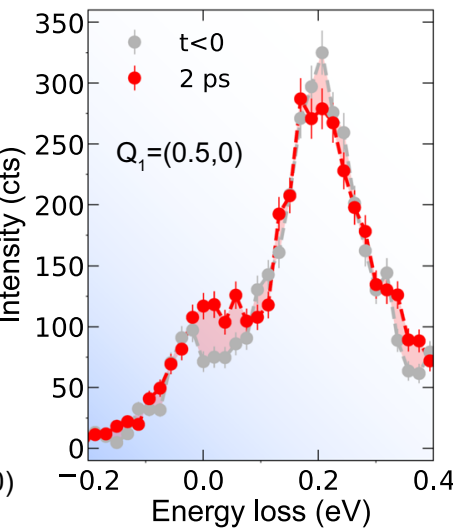

C

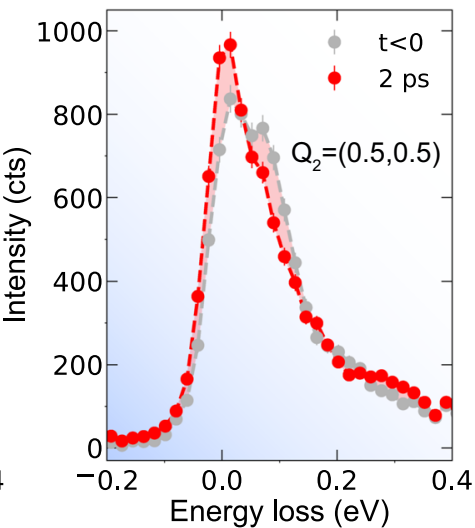

Fig. 3 Driven magnetic excitations in $\mathbf{S r}_{\mathbf{2}} \mathbf{I r O} \mathbf{O}_{\mathbf{4}}$. a Dispersion of the in-plane pseudospin excitation spectrum in $\mathrm{Sr}_{2} \mid \mathrm{rO} \mathrm{O}_{4}$ as function of momentum transfer $q$. Black circles correspond to momentum transfer points shown in the other two panels. b, c Ir L-edge (11.215 keV) time-resolved resonant inelastic X-ray scattering (trRIXS) snapshots of the pseudospin excitations excited with a $620 \mathrm{meV}$ pump pulse at negative time delay $t$ (gray) and after 2 picoseconds (red) at momentum transfer $\mathbf{Q}_{1}=(0.5,0)$ and $\mathbf{Q}_{2}=(0.5,0.5)$ reciprocal lattice units (r.l.u.). The intensity is measured in counts (cts), while error bars represent Poisson counting uncertainties. The light-induced spectral weight change is highlighted by a red shading. Adapted with author's permission from ref. 61 .

\section{An evolving theoretical framework}

A fundamental need for current and future trRIXS research lies in calculating the cross-section and predicting the many-body response of photoexcited quantum materials. Unlike other spectroscopies (e.g., trARPES, and non-resonant light/x-ray scattering $)^{67-70}$, the RIXS process involves a four-time correlation function, owing to the presence of the resonant intermediate state $^{71,72}$. Although this complexity entails modeling and numerical challenges, it is precisely the intermediate state dynamics which makes RIXS sensitive to a wide variety of collective excitations. Moreover, these excitations encode high-order correlations beyond the linear response, and thus play a crucial role in emergent phenomena with strong quantum fluctuations.
If the intermediate state is assumed to last for a negligibly short time (ultrashort core-hole lifetime (UCL) approximation), the dominant contribution to the equilibrium RIXS spectrum comes from the dynamical structure factors ${ }^{32,73-76}$. For this reason, early theories of trRIXS focused on calculating charge and spin structure factors in correlated electron models driven out of equilibrium ${ }^{68,77}$. For example, an early simulation of a onedimensional Mott insulator excited by a realistic below-gap pump pulse found evidence of light-induced Floquet replicas in both the spin and charge response. Different from an ideal Floquet picture, these light-engineered excitations persisted and evolved after the pump pulse ${ }^{68}$, thus indicating that light pulses are conducive to emergent Floquet dynamics at finite momentum. 
a

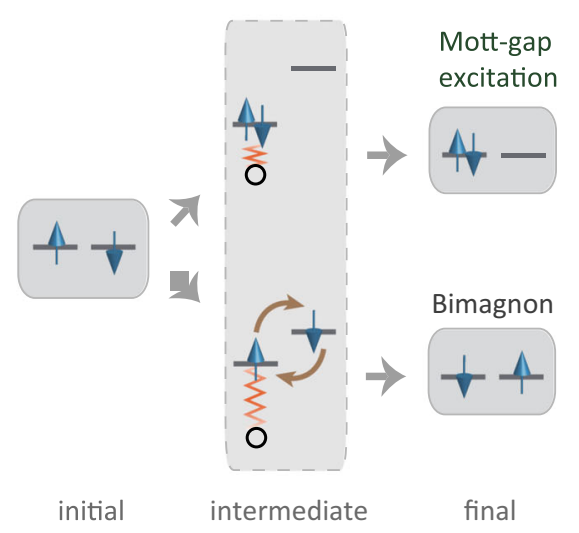

b

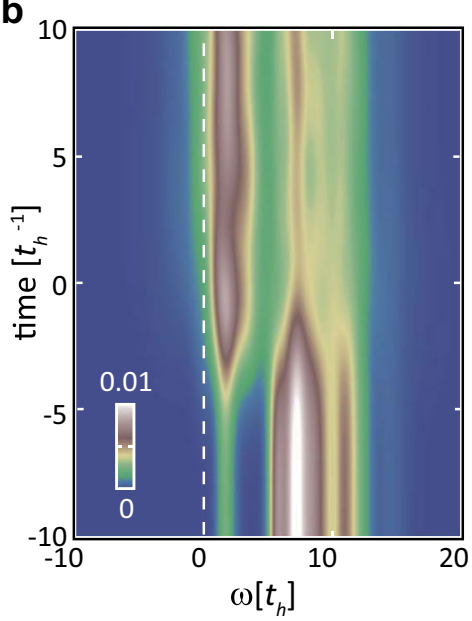

C

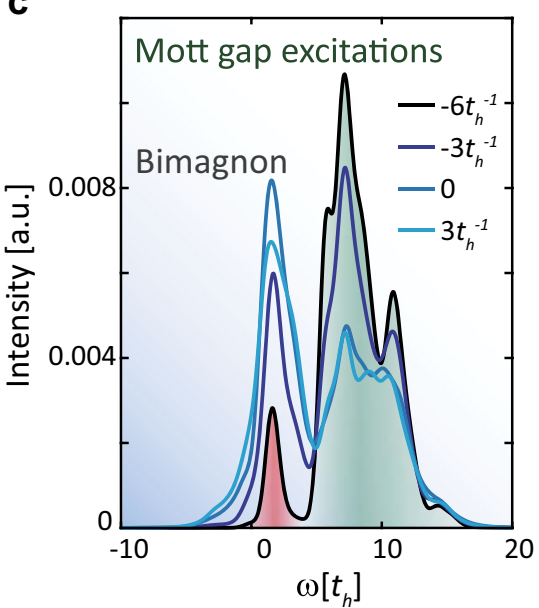

Fig. 4 Theoretical simulation of the time-resolved resonant inelastic X-ray scattering (trRIXS) spectrum in a pumped Mott insulator. a Dominant excitations in the $K$-edge (indirect) trRIXS spectrum of the single-band Hubbard model in two dimensions. The open circle represents the core hole, whereas the red zig-zag line is its Coulomb interaction with the valence electron spins (up down arrows). $\mathbf{b}$ Evolution of the trRIXS spectrum obtained through exact diagonalization for momentum transfer $\mathbf{Q}=(1 / 2,1 / 6)$ reciprocal lattice units (r.l.u.) and incident photon energy tuned to the maximum of the X-ray absorption spectrum. $\omega$ denotes the energy loss axis measured in units of hopping energy $\left(t_{h}\right)$. c Selected trRIXS spectra at different times before and after the pump. Bimagnon features are located at $\hbar \omega \sim 3$, whereas the Mott-gap excitation continuum is peaked at $\hbar \omega \sim U=8 t_{h}$. $U$ is the onsite Coulomb repulsion, whereas $J=4 t_{h}^{2} / U$ is the exchange energy. Adapted with permission from ref. ${ }^{72}$. Copyrighted by the American Physical Society.

Although these results constitute an exciting starting point, the UCL approximation ignores physical processes faster than the timescale of the core-hole lifetime (longer than $2 \mathrm{fs}$ at the $\mathrm{Cu} \mathrm{L}$ edge $^{32,78}$ ) as well as high-order correlations ${ }^{79}$. Therefore, a simple calculation of the structure factors may not capture the full trRIXS spectrum and miss higher order spectral features such as bimagnons and $d d$ excitations. In order to compute the full trRIXS cross-section, one needs to explicitly model the resonant probe process and account for the finite intermediate state lifetime, leading to a computational complexity $O\left(N_{\mathrm{t}}^{4}\right)$, where $N_{\mathrm{t}}$ is the number of evolution steps ${ }^{71,72}$.

Figure 4 shows the numerically calculated trRIXS spectrum of a two-dimensional Hubbard model probed through an indirect scattering process (e.g., $\mathrm{Cu} \mathrm{K}$-edge) and explicitly accounting for intermediate state effects. Here, excitations are generated through interactions between the valence electrons and the core hole during the intermediate state ${ }^{72}$. Unlike the structure factors obtained within the UCL approximation, the trRIXS cross-section contains high-order excitations including the bimagnon at energy $\sim 3 J\left(J=4 t_{\mathrm{h}}^{2} / U\right.$ is the spin-exchange interaction, $t_{\mathrm{h}}$ is the hopping amplitude, and $U$ is the onsite Coulomb repulsion), in addition to Mott-gap excitations. After being driven by a pump resonant with the Mott gap, spectral weight gets transferred from the Mott peak to in-gap excitations and bimagnons. In this case, the visibility of Floquet replicas of collective excitations is reduced when compared with ref. ${ }^{68,71}$, mainly due to the shorter Floquet state lifetime in the presence of electron-electron interactions. The predicted in-gap spectral weight transfer resembles the quasielastic scattering intensity enhancement observed in the iridate $L$-edge trRIXS spectra (see Fig. 3), and could be measured in future $K$-edge experiments on gapped correlated materials.

Intermediate state effects are not only required to capture richer physics in trRIXS simulations, but will also guide the interpretation of new types of inelastic scattering experiments. RIXS spectra collected while slightly detuning the incident X-ray pulse energy away from resonance can be used to selectively enhance the intensity of excitations involving specific intermediate states ${ }^{72,80}$ and to extract information about momentum-resolved electron-phonon coupling of well-isolated modes ${ }^{78,81,82}$.
Detuning experiments, in tandem with microscopic calculations, will be particularly impactful in interrogating the electron-phonon coupling in photoexcited charge density waves ${ }^{11,49,53}$, lightinduced ferroelectricity ${ }^{13}$, and superconductivity ${ }^{83}$.

In summary, advances in trRIXS experiments are accompanied by a steadily evolving theoretical framework aimed at understanding the observed light-induced dynamics. Current computational capabilites and state-of-the-art algorithms allow for performing accurate and predictive nonequilibrium simulations of the trRIXS spectrum in photoexcited correlated electron systems. In the future, we expect trRIXS theory to play an ever important role not just in interpreting time-resolved scattering experiments, but in leading the field towards new discoveries.

\section{A new scientific opportunity}

As shown in previous sections, trRIXS is rapidly growing into a major spectroscopy of light-driven quantum materials, and its future developments are critically tied to the pace of technological advances at XFEL facilities. Owing to the small inelastic cross sections, trRIXS will benefit from a dramatic increase in the average XFEL spectral brightness from $10^{20}$ (LCLS) to $10^{24}-10^{25}$ photons s $\mathrm{mm}^{-1} \mathrm{mrad}^{-2}(0.1 \% \text { bandwidth })^{-1}$ at $1 \mathrm{keV}^{30,84}$. This brightness enhancement will be mainly achieved through increased repetition rates, e.g., at both the LCLS-II and the European XFEL, and will lead to order-of-magnitude improvements of the signal-to-noise ratio. Furthermore, higher energy resolution (especially in the soft $\mathrm{X}$-ray regime) will enable observing low-energy collective fluctuations, such as phonons and spin waves. At the time of this writing, large spectrometers are in construction at both the LCLS-II (NEH2.2/q-RIXS instrument) ${ }^{84}$ and the European XFEL (hRIXS instrument). The target resolving power at $1 \mathrm{keV}$ would be of order $3.0 \times 10^{4}(\sim 0.03 \mathrm{eV}$ resolution), thus implying a $20 \times$ improvement with respect to the data shown in Fig. $2^{85}$. Other experimental endstations with different features are being developed at the Trieste Free Electron laser Radiation for Multidisciplinary Investigations (FERMI/Italy), the Pohang Accelerator Laboratory X-ray Free Electron Laser (PAL-XFEL/S. Korea), and the Switzerland's X-ray free-electron laser (SwissFEL). These enhanced capabilities open up a new frontier for the 
a

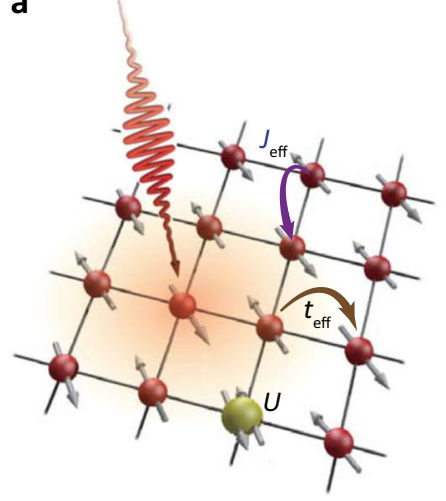

b

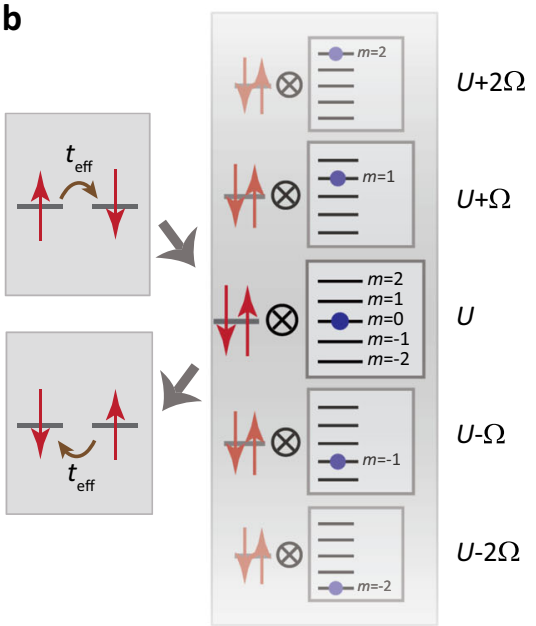

C

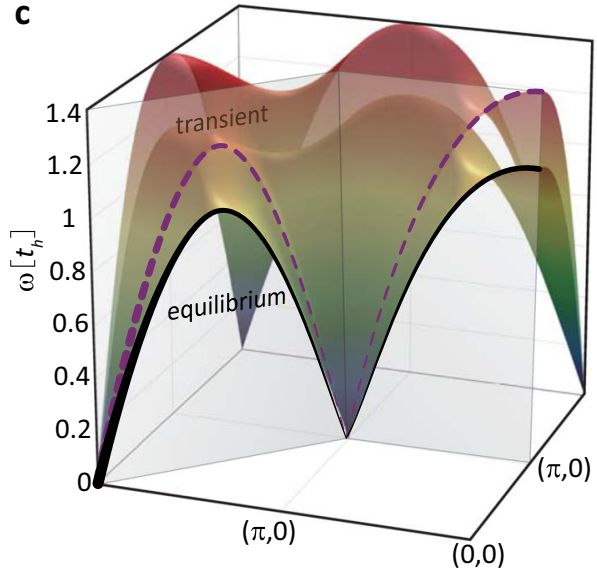

Fig. 5 Ultrafast manipulation of finite-momentum spin waves. a Ultrafast pulses can be used to directly manipulate magnetic excitations in quantum materials through a direct modulation of the effective exchange interactions $J_{\text {eff }} \mathbf{b}$ A possible mechanism consists of dressing the intermediate doubly occupied state with the pump field, which is lifted (lowered) in energy by an amount proportional to the number of virtually absorbed (emitted) pump photons. $U$ is the onsite Coulomb repulsion, $t_{\text {eff }}$ the effective hopping amplitude, $\Omega$ the pump photon energy and $m$ the Floquet index. $\mathbf{c}$ As the exchange interactions are modulated, the spin wave dispersion is expected to shift in energy $(\omega)$ across the entire Brillouin zone. A cross-section cut (gray shaded planes) along two high-symmetry directions is included for clarity.

investigation of nonequilibrium spin, charge and orbital dynamics in strongly correlated and topologically non-trivial materials.

A tantalizing application of trRIXS is the study of spin excitations in light-driven quantum materials. Multiple theoretical studies have proposed that spin-exchange interactions can be controlled by renormalizing the effective Hamiltonian interactions through a periodic drive (see Fig. $5 \mathrm{a}$ ), in the spirit of the so-called "Floquet-engineering"86-89. In this idealized scheme, photons dress the intermediate electronic states of the exchange (or superexchange) process, leading to an effective energy scale $J_{\text {eff }}$ (see Fig. 5b). Although the parameter renormalization is rigorous only for an infinitely-long periodic pump, it could also be achieved with an ultrashort laser pulse ${ }^{70}$. Alternatively, the spin excitations could be dynamically altered through other protocols, e.g., by distorting the lattice via a nonlinear phonon coupling 90,91 . Once $J_{\text {eff }}$ is modified by the pump, trRIXS measurements will interrogate changes in the dispersion, linewidth and spectral weight of the spin fluctuation spectrum (see Fig. 5c). A measurement of the dispersion allows us to disentangle renormalization effects of multiple coexisting exchange interactions ${ }^{92}$, which cannot be discerned from the bimagnon peak in the optical Raman spectrum. Furthermore, a lineshape analysis throughout the Brillouin zone enables exploring excitations beyond magnons, e.g., incoherent fluctuations in geometrically frustrated lattices ${ }^{93-96}$. As the experimental energy resolution will be of order $\sim 0.03 \mathrm{eV}$ in the near future 84,85 , this technique will be particularly effective for studying materials with relatively large exchange scales, such as cuprates, iridates and certain nickelates, where the spin excitations disperse over energies larger than $0.1 \mathrm{eV}$.

In addition to spin fluctuations, trRIXS will play as well a pivotal role as a probe of ultrafast charge and orbital dynamics. We envision here two promising research directions involving the charge sector of low-dimensional quantum materials, namely the time-resolved investigation of fractionalized excitations, and the search for new photoinduced condensation phenomena. In one dimension, electrons cannot propagate freely, but instead displace their neighbors due to electron-electron interactions. This leads to a breakdown ("fractionalization") of the electron into a variety of collective excitations propagating with different velocities ${ }^{97}$. trRIXS provides an opportunity to study these fundamental phenomena in real time by exciting a transient particlehole plasma with a high photon energy pump and directly observing the time-dependent behavior of the collective modes contained in the transient RIXS spectrum. First experiments could focus on fractionalization in $\mathrm{Sr}_{2} \mathrm{CuO}_{3}{ }^{98}$ or $\mathrm{CaCu}_{2} \mathrm{O}_{3}{ }^{99}$, and on manipulating their excitations by dynamically tuning the balance between spin-orbit coupling and crystal field ${ }^{100}$. Similarly, trRIXS experiments in photoexcited Mott insulators could reveal the fingerprints of new condensation phenomena, such as $\eta$ pairing ${ }^{101,102}$ and dynamical $p$-wave superconductivity ${ }^{103}$. The $\eta$ paired phase is a superfluid of doubly occupied electronic states carrying finite-momentum $\mathbf{Q}_{\eta}$ and arising from a broken $\mathrm{SU}(2)$ symmetry of the Hubbard Hamiltonian. As the $\eta$-pairing is an eigenstate, but not necessarily a ground state, pump light pulses open the possibility of stabilizing this yet unobserved phase $\mathrm{e}^{104-110}$. A periodic pump is theoretically predicted to enhance pairing correlations and establish true off-diagonal long-range order by dynamically renormalizing the onsite Coulomb repulsion ${ }^{108}$. trRIXS would then search for $\eta$-pairing signatures in the finitemomentum pairing susceptibility ${ }^{111}$, namely a divergent quasielastic structure factor at $\mathbf{Q}_{\eta}$ and a triplet of collective modes at energies $\hbar \omega=0, \pm(U-2 \mu)$ ( $\mu$ being the chemical potential $)^{102}$. Beyond $\eta$-pairing, resonantly driving orbital degrees of freedom in doped Mott insulators has also been proposed as a route to dynamically stabilize $p$-wave superconductivity ${ }^{103}$.

The search for new light-driven phenomena in materials dominated by local electronic correlations also calls for the development of more advanced spectroscopic methods. One such approach is trRIXS interferometry. Thanks to the local nature of the intermediate core holes and the intrinsic coherence of the scattering process, the RIXS signal can indeed exhibit interference among different intermediate states ${ }^{112}$. In the dimerized spinorbit coupled insulator $\mathrm{Ba}_{3} \mathrm{CeIr}_{2} \mathrm{O}_{9}{ }^{113}$, the intermediate state involves a coherent superposition of a single core hole on either of the two atoms in the dimer. This leads to a $\cos ^{2}(\mathbf{Q} \cdot \mathbf{d} / 2)$ modulation of the RIXS intensity in momentum space ( $\mathbf{d}$ being the intradimer distance). Importantly, the interference pattern varies in amplitude and phase depending on the symmetry of the excited-state wavefunction ${ }^{113}$, thus providing an interferometric 
a

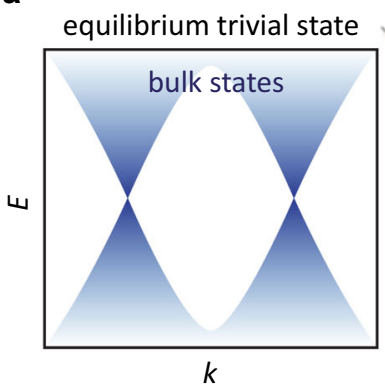

b

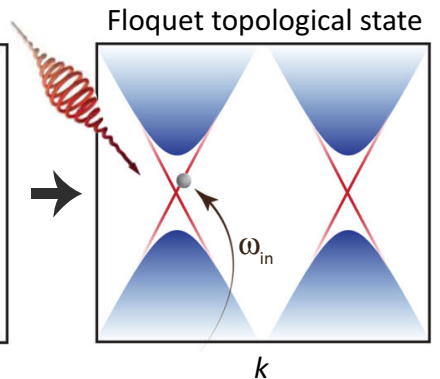

C

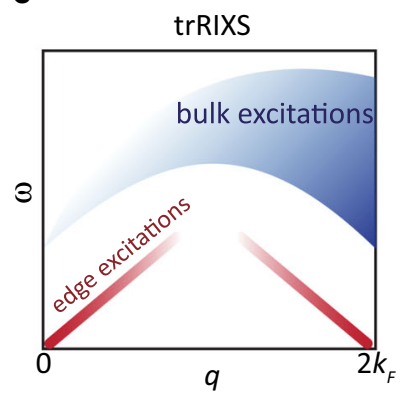

Fig. 6 Generation and detection of topological collective modes. a Circularly polarized pump pulses can drive bulk Dirac carriers (blue) and $\mathbf{b}$ open a gap at the Dirac points by breaking time-reversal symmetry. $E$ and $k$ denote energy and momentum of the band structure, respectively, whereas $\omega_{\text {in }}$ stands for the incoming X-ray photon energy. In addition to the light-induced Floquet bandgap, the system exhibits chiral edge states (red) which give rise to dispersing collective modes in the time-resolved resonant inelastic X-ray scattering (trRIXS) spectrum c. These collective modes are linearly dispersing and only present at overlap with the pump excitation, unlike the particle-hole continuum owing to the bulk states. $\omega$ and $q$ indicate energy loss and momentum transfer, whereas $k_{\mathrm{F}}$ represents the Fermi wavevector.

measurement of the local atomic orbitals. By sampling multiple interference fringes in the hard X-ray regime (e.g., Ir $L$-edge, and $\mathrm{Cu} K$-edges), it would be possible to exquisitely resolve transient changes to the local electronic structure down to the picometer level with energy selectivity. Not just limited to dimerized compounds, this technique could likewise be applied to study nonequilibrium dynamics of confined electrons along one or two directions, such as in ladder compounds ${ }^{114}$ or layered cuprates ${ }^{83}$ and nickelates ${ }^{115,116}$.

In a very different context, confined electronic motion is also a defining property of edge states in light-induced topological phases, which could be revealed by trRIXS experiments ${ }^{80}$. Circularly polarized laser pulses have been shown to break timereversal symmetry and induce transient states with non-trivial Chern numbers ${ }^{21,117-119}$. An intriguing application of this experimental approach is the creation of tunable Floquet topological insulators (FTIs), in which topology can be manipulated by varying pump amplitude, energy, and polarization ${ }^{120-124}$. As shown in Fig. 6a, a possible route for creating a FTI in two dimensions starts from a material with bulk massless Dirac fermions. The circularly polarized pump induces a gap opening at the Dirac point and chiral edge modes at the sample boundary, which disperse across the light-induced bandgap (see Fig. 6b). Detecting these edge states entails probing either the transient band structure with trARPES, or their collective modes in the dynamic structure factor through trRIXS, depending on the experimental constraints. However, trRIXS offers a crucial advantage. By resonantly tuning the incident photon energy to transitions from core states into the light-induced bandgap, this technique can boost the visibility of the topological edge states over the bulk signal, and hence distinguish their dispersion from other bulk collective modes ${ }^{80}$ (see Fig. 6c). Future developments in nano-trRIXS may enable the direct spatial imaging of edge state dynamics and, thus, further increase their visibility over bulk excitations (although these experiments would require a special handling of X-ray irradiation effects). Finally, an additional area of interest (particularly for hard X-ray trRIXS) is the light-control of candidate topological superconductors under high pressures $^{125-128}$, which are inaccessible to photoemission experiments.

This short, and by no means complete, array of examples underscores how trRIXS measurements, alongside new theoretical methods, will play an essential role in detecting and understanding new dynamic phenomena in light-controlled quantum materials. Increased XFEL beamtime availability, X-ray brightness and spectrometer performance will enable more sophisticated trRIXS experiments with higher energy resolution and polarization control. The possibilities opened by these advances are difficult to grasp, but are certainly positioning trRIXS to be on the leading edge of a decade of discovery.

Received: 22 July 2020; Accepted: 16 September 2020; Published online: 19 October 2020

\section{References}

1. Keimer, B. \& Moore, J. E. The physics of quantum materials. Nat. Phys. 13, 1045-1055 (2017).

2. Stanciu, C. D. et al. All-optical magnetic recording with circularly polarized light. Phys. Rev. Lett. 99, 047601 (2007).

3. Nova, T. F. et al. An effective magnetic field from optically driven phonons. Nat. Phys. 13, 132-136 (2016).

4. Afanasiev, D. et al. Light-driven ultrafast phonomagnetism. arXiv (2019). 1912.01938v1.

5. Mikhaylovskiy, R. V. et al. Ultrafast optical modification of exchange interactions in iron oxides. Nat. Commun. 6, 8190 (2015).

6. Disa, A. S. et al. Polarizing an antiferromagnet by optical engineering of the crystal field. Nat. Phys. 16, 937-941 (2020).

7. Schmitt, F. et al. Transient electronic structure and melting of a charge density wave in $\mathrm{TbTe}_{3}$. Science 321, 1649-1652 (2008).

8. Perfetti, L. et al. Femtosecond dynamics of electronic states in the Mott insulator $1 \mathrm{~T}-\mathrm{TaS}_{2}$ by time resolved photoelectron spectroscopy. N. J. Phys. 10, 053019 (2008).

9. Rohwer, T. et al. Collapse of long-range charge order tracked by time-resolved photoemission at high momenta. Nature 471, 490-493 (2011).

10. Kim, K. W. et al. Ultrafast transient generation of spin-density- wave order in the normal state of $\mathrm{BaFe}_{2} \mathrm{As}_{2}$ driven by coherent lattice vibrations. Nat. Mater. 11, 497-501 (2012).

11. Lee, W. S. et al. Phase fluctuations and the absence of topological defects in a photo-excited charge-ordered nickelate. Nat. Commun. 3, 257-6 (2012).

12. Kubacka, T. et al. Large-amplitude spin dynamics driven by a THz pulse in resonance with an electromagnon. Science 343, 1333-1336 (2014).

13. Nova, T. F., Disa, A. S., Fechner, M. \& Cavalleri, A. Metastable ferroelectricity in optically strained $\mathrm{SrTiO}_{3}$. Science 364, 1075-1079 (2019).

14. Li, X. et al. Terahertz field-induced ferroelectricity in quantum paraelectric $\mathrm{SrTiO}_{3}$. Science 364, 1079-1082 (2019).

15. Fausti, D. et al. Light-induced superconductivity in a stripe-ordered cuprate. Science 331, 189-191 (2011). Discovery of light-induced superconductivity by resonantly driving a phonon mode in a chargeordered cuprate.

16. $\mathrm{Hu}, \mathrm{W}$. et al. Optically enhanced coherent transport in $\mathrm{YBa}_{2} \mathrm{Cu}_{3} \mathrm{O}_{6.5}$ by ultrafast redistribution of interlayer coupling. Nat. Mater. 13, 705-711 (2014). 
17. Kaiser, S. et al. Optically induced coherent transport far above $\mathrm{Tc}$ in underdoped $\mathrm{YBa}_{2} \mathrm{Cu}_{3} \mathrm{O}_{6+\delta}$. Phys. Rev. B 89, 184516 (2014).

18. Mitrano, M. et al. Possible light-induced superconductivity in $\mathrm{K}_{3} \mathrm{C}_{60}$ at high temperature. Nature 530, 461-464 (2016).

19. Wang, Y. H., Steinberg, H., Jarillo-Herrero, P. \& Gedik, N. Observation of Floquet-Bloch States on the Surface of a Topological Insulator. Science $\mathbf{3 4 2}$ 453-457 (2013). Discovery of Floquet-Bloch bands induced by ultrafast midinfrared pulses.

20. Mahmood, F. et al. Selective scattering between Floquet-Bloch and Volkov states in a topological insulator. Nat. Phys. 12, 306-310 (2016).

21. McIver, J. W. et al. Light-induced anomalous Hall effect in graphene. Nat. Phys. 16, 38-41 (2019).

22. Siders, C. W. et al. Detection of nonthermal melting by ultrafast X-ray diffraction. Science 286, 1340-1342 (1999).

23. Fritz, D. M. et al. Ultrafast bond softening in bismuth: mapping a solid's interatomic potential with X-rays. Science 315, 633-636 (2007).

24. Sokolowski-Tinten, K. et al. Femtosecond X-ray measurement of coherent lattice vibrations near the Lindemann stability limit. Nature 422, 287-289 (2003).

25. Gedik, N., Yang, D., Logvenov, G., Bozovic, I. \& Zewail, A. Nonequilibrium phase transitions in cuprates observed by ultrafast electron crystallography. Science 316, 425 (2007).

26. Smallwood, C. L. et al. Tracking cooper pairs in a cuprate superconductor by ultrafast angle-resolved photoemission. Science 336, 1137-1139 (2012).

27. Ulbricht, R., Hendry, E., Shan, J., Heinz, T. F. \& Bonn, M. Carrier dynamics in semiconductors studied with time-resolved terahertz spectroscopy. Rev. Mod. Phys. 83, 543-586 (2011).

28. Giannetti, C. et al. Ultrafast optical spectroscopy of strongly correlated materials and high-temperature superconductors: a non-equilibrium approach. Adv. Phys. 65, 58-238 (2016).

29. Nicoletti, D. \& Cavalleri, A. Nonlinear light-matter interaction at terahertz frequencies. Adv. Opt. Photonics 8, 401 (2016).

30. Bostedt, C. et al. Linac Coherent Light Source: The first five years. Rev. Mod. Phys. 88, 015007 (2016).

31. Cao, Y. et al. Ultrafast dynamics of spin and orbital correlations in quantum materials: an energy-and momentum-resolved perspective. Philos. Trans. $R$. Soc. A 377, 20170480 (2019).

32. Ament, L. J. P., van Veenendaal, M., Devereaux, T. P., Hill, J. P. \& van den Brink, J. Resonant inelastic $\mathrm{x}$-ray scattering studies of elementary excitations. Rev. Mod. Phys. 83, 705-767 (2011). Authoritative review article discussing theoretical and experimental basics of equilibrium RIXS, including early experimental work.

33. Haverkort, M. W. Theory of resonant inelastic x-ray scattering by collective magnetic excitations. Phys. Rev. Lett. 105, 167404 (2010).

34. Wernet, P. et al. Orbital-specific mapping of the ligand exchange dynamics of $\mathrm{Fe}(\mathrm{CO})_{5}$ in solution. Nature 520, 78-81 (2015).

35. Jay, R. M. et al. Disentangling transient charge density and metal-ligand covalency in photoexcited ferricyanide with femtosecond resonant inelastic soft X-ray scattering. J. Phys. Chem. Lett. 9, 3538-3543 (2018).

36. Lundberg, M. \& Wernet, P. Resonant Inelastic X-ray Scattering (RIXS) Studies in Chemistry: Present and Future. 1-52 (Springer International Publishing, Cham, 2019).

37. Tranquada, J. M. et al. Quantum magnetic excitations from stripes in copper oxide superconductors. Nature 429, 534-538 (2004).

38. Abbamonte, P. et al. Spatially modulated 'Mottness' in $\mathrm{La}_{2-x} \mathrm{Ba}_{x} \mathrm{CuO}_{4}$. Nat. Phys. 1, 155-158 (2005).

39. Ghiringhelli, G. et al. Long-range incommensurate charge fluctuations in (Y, $\mathrm{Nd}) \mathrm{Ba}_{2} \mathrm{Cu}_{3} \mathrm{O}_{6+x}$. Science 337, 821-825 (2012).

40. Comin, R. et al. Charge order driven by Fermi-Arc instability in $\mathrm{Bi}_{2} \mathrm{Sr}_{2-x} \mathrm{La}_{x}$ $\mathrm{CuO}_{6+\delta}$. Science 343, 390-392 (2014).

41. da Silva Neto, E. H. et al. Ubiquitous interplay between charge ordering and high-temperature superconductivity in cuprates. Science 343, 393-396 (2014).

42. Huang, E. W. et al. Numerical evidence of fluctuating stripes in the normal state of high-Tc cuprate superconductors. Science 358, 1161-1164 (2017).

43. Zheng, B.-X. et al. Stripe order in the underdoped region of the twodimensional Hubbard model. Science 358, 1155-1160 (2017).

44. Chang, J. et al. Direct observation of competition between superconductivity and charge density wave order in $\mathrm{YBa}_{2} \mathrm{Cu}_{3} \mathrm{O}_{6.67}$. Nature Physics 8, 871-876 (2012).

45. Jiang, H.-C. \& Devereaux, T. P. Superconductivity in the doped Hubbard model and its interplay with next-nearest hopping t. Science 365, 1424-1428 (2019).

46. Nicoletti, D. et al. Optically induced superconductivity in striped $\mathrm{La}_{2-x} \mathrm{Ba}_{x} \mathrm{CuO}_{4}$ by polarization-selective excitation in the near infrared. Phys. Rev. B 90, 100503 (2014).
47. Först, $\mathrm{M}$. et al. Melting of charge stripes in vibrationally driven $\mathrm{La}_{1.875} \mathrm{Ba}_{0.125} \mathrm{CuO}_{4}$ : assessing the respective roles of electronic and lattice order in frustrated superconductors. Phys. Rev. Lett. 112, 157002 (2014).

48. Först, $\mathrm{M}$. et al. Femtosecond $\mathrm{x}$ rays link melting of charge-density wave correlations and light-enhanced coherent transport in $\mathrm{YBa}_{2} \mathrm{Cu}_{3} \mathrm{O}_{6.6}$. Phys. Rev. B 90, 184514 (2014).

49. Wandel, S. et al. Light-enhanced charge density wave coherence in a hightemperature superconductor. arXiv:2003.04224 (2020).

50. Mitrano, M. et al. Ultrafast time-resolved $\mathrm{x}$-ray scattering reveals diffusive charge order dynamics in $\mathrm{La}_{2-\mathrm{x}} \mathrm{Ba}_{\mathrm{x}} \mathrm{CuO}_{4}$. Sci. Adv. 5, eaax3346 (2019). Observation of diffusive charge order dynamics in a cuprate superconductor with soft X-ray trRIXS.

51. Mitrano, M. et al. Evidence for photoinduced sliding of the charge-order condensate in $\mathrm{La}_{1.875} \mathrm{Ba}_{0.125} \mathrm{CuO}_{4}$. Phys. Rev. B 100, 205125 (2019).

52. Cremin, K. A. et al. Photoenhanced metastable c-axis electrodynamics in stripe-ordered cuprate $\mathrm{La}_{1.885} \mathrm{Ba}_{0.115} \mathrm{CuO}_{4}$. Proc. Natl Acad. Sci. 116, 19875-19879 (2019)

53. Huber, T. et al. Coherent structural dynamics of a prototypical chargedensity-wave-to-metal transition. Phys. Rev. Lett. 113, 026401 (2014).

54. Arpaia, R. et al. Dynamical charge density fluctuations pervading the phase diagram of a Cu-based high- $\mathrm{T}_{c}$ superconductor. Science 365, 906-910 (2019).

55. Scalapino, D., Loh Jr, E. \& Hirsch, J. D-wave pairing near a spin-density-wave instability. Phys. Rev. B 34, 8190 (1986).

56. Gros, C., Joynt, R. \& Rice, T. Superconducting instability in the large-U limit of the two-dimensional Hubbard model. Z. Phys. B Condens. Matter 68, 425-432 (1987).

57. Kotliar, G. \& Liu, J. Superexchange mechanism and d-wave superconductivity. Phys. Rev. B 38, 5142 (1988).

58. Tsuei, C. \& Kirtley, J. Pairing symmetry in cuprate superconductors. Rev. Modern Phys. 72, 969 (2000).

59. Scalapino, D. J. A common thread: the pairing interaction for unconventional superconductors. Rev. Mod. Phys. 84, 1383-1417 (2012).

60. Maier, T. A. et al. Pairing in a dry Fermi sea. Nat. Commun. 7, 1-6 (2016),

61. Dean, M.P. et al. Ultrafast energy-and momentum-resolved dynamics of magnetic correlations in the photo-doped Mott insulator $\mathrm{Sr}_{2} \mathrm{IrO}_{4}$. Nat. Mater. 15, 601-605 (2016). First hard X-ray trRIXS investigation of lightdriven pseudospins in a strongly-correlated material.

62. Mazzone, D. et al. Trapped transient magnons in the gapped antiferromagnet $\mathrm{Sr}_{3} \mathrm{Ir}_{2} \mathrm{O}_{7}$. arXiv:2002.07301 (2020).

63. Wang, F. \& Senthil, T. Twisted Hubbard model for $\mathrm{Sr}_{2} \mathrm{IrO}_{4}$ : magnetism and possible high temperature superconductivity. Phys. Rev. Lett. 106, 136402 (2011).

64. Kim, Y. K. et al. Fermi arcs in a doped pseudospin-1/2 heisenberg antiferromagnet. Science 345, 187-190 (2014).

65. Parchenko, S. et al. Orbital dynamics during an ultrafast insulator to metal transition. Phys. Rev. Res. 2, 023110 (2020).

66. Dell'Angela, M. et al. Extreme ultraviolet resonant inelastic X-ray scattering (RIXS) at a seeded free-electron laser. Sci. Rep. 6, 38796 (2016).

67. Freericks, J. K., Krishnamurthy, H. R. \& Pruschke, T. Theoretical description of time-resolved photoemission spectroscopy: application to pump-probe experiments. Phys. Rev. Lett. 102, 136401 (2009).

68. Wang, Y., Claassen, M., Moritz, B. \& Devereaux, T. Producing coherent excitations in pumped Mott antiferromagnetic insulators. Phys. Rev. B 96, 235142 (2017)

69. Freericks, J., Matveev, O., Shvaika, A. \& Devereaux, T. Nonresonant pump/ probe electronic Raman scattering within nonequilibrium dynamical meanfield theory. In Ultrafast Bandgap Photonics III, vol. 10638, 1063807 (International Society for Optics and Photonics, 2018).

70. Wang, Y., Devereaux, T. P. \& Chen, C.-C. Theory of time-resolved Raman scattering in correlated systems: ultrafast engineering of spin dynamics and detection of thermalization. Phys. Rev. B 98, 245106 (2018).

71. Chen, Y. et al. Theory for time-resolved resonant inelastic $\mathrm{x}$-ray scattering. Phys. Rev. B 99, 104306 (2019). This paper provides a comprehensive theoretical derivation of the trRIXS cross-section.

72. Wang, Y., Chen, Y., Jia, C., Moritz, B. \& Devereaux, T. P. Time-resolved resonant inelasticx-ray scattering in a pumped Mott insulator. Phys. Rev. B 101,165126 (2020). This paper reports the first numerical simulation of the indirect trRIXS spectrum of a correlated electron system.

73. Van Den Brink, J. The theory of indirect resonant inelastic X-ray scattering on magnons. Europhys. Lett. 80, 47003 (2007).

74. Ament, L. J., Forte, F. \& van den Brink, J. Ultrashort lifetime expansion for indirect resonant inelastic x-ray scattering. Phys. Rev. B 75, 115118 (2007).

75. Jia, C., Wohlfeld, K., Wang, Y., Moritz, B. \& Devereaux, T. P. Using RIXS to uncover elementary charge and spin excitations. Phys. Rev. X 6, 021020 (2016).

76. Ament, L. J. P., Ghiringhelli, G., Sala, M. M., Braicovich, L. \& van den Brink, J. Theoretical Demonstration of How the Dispersion of Magnetic Excitations in 
Cuprate Compounds can be Determined Using Resonant Inelastic X-Ray Scattering. Phys. Rev. Lett. 103, 117003 (2009).

77. Paeckel, S. et al. Detecting superconductivity out of equilibrium. Phys. Rev. B 101, 180507 (2020).

78. Rossi, M. et al. Experimental determination of momentum-resolved electronphonon coupling. Phys. Rev. Lett. 123, 027001 (2019).

79. Tohyama, T. \& Tsutsui, K. Spectral weight of resonant inelastic x-ray scattering in doped cuprates: effect of core-hole lifetime. Int. J. Modern Phys. B 32, 1840017 (2018)

80. Chen, Y., Wang, Y., Claassen, M., Moritz, B. \& Devereaux, T. P. Observing photo-induced chiral edge states of graphene nanoribbons in pump-probe spectroscopies. arXiv:2005.00684 (2020).

81. Ament, L., Van Veenendaal, M. \& Van Den Brink, J. Determining the electron-phonon coupling strength from resonant inelastic $\mathrm{x}$-ray scattering at transition metal L-edges. EPL 95, 27008 (2011).

82. Geondzhian, A. \& Gilmore, K. Generalization of the Franck-Condon model for phonon excitations by resonant inelastic x-ray scattering. Phys. Rev. B 101, 214307 (2020).

83. Mankowsky, R. et al. Nonlinear lattice dynamics as a basis for enhanced superconductivity in $\mathrm{YBa}_{2} \mathrm{Cu}_{3} \mathrm{O}_{6.5}$. Nature 516, 71-73 (2014).

84. Dunne, M. Lcls strategic facility development plan (2019). https://lcls.slac stanford.edu/sites/lcls.slac.stanford.edu/files/LCLS_Strategic_Development_ Plan.pdf.

85. Dunne, M. X-ray free-electron lasers light up materials science. Nat. Rev. Mater. 3, 290-292 (2018)

86. Mentink, J. H. \& Eckstein, M. Ultrafast quenching of the exchange interaction in a Mott insulator. Phys. Rev. Lett. 113, 057201 (2014).

87. Mentink, J. H., Balzer, K. \& Eckstein, M. Ultrafast and reversible control of the exchange interaction in Mott insulators. Nat. Commun. 6, 1-8 (2015). Theoretical proposal for the transient manipulation of the exchange interaction via Floquet engineering of the intermediate electronic state.

88. Chaudhary, S., Hsieh, D. \& Refael, G. Orbital floquet engineering of exchange interactions in magnetic materials. Phys. Rev. B 100, 220403 (2019).

89. Walldorf, N., Kennes, D. M., Paaske, J. \& Millis, A. J. The antiferromagnetic phase of the Floquet-driven Hubbard model. Phys. Rev. B 100, 121110 (2019).

90. Först, M. et al. Nonlinear phononics as an ultrafast route to lattice control. Nat. Phys. 7, 854-856 (2011).

91. Subedi, A., Cavalleri, A. \& Georges, A. Theory of nonlinear phononics for coherent light control of solids. Phys. Rev. B 89, 220301 (2014).

92. Peng, Y. Y. et al. Influence of apical oxygen on the extent of in-plane exchange interaction in cuprate superconductors. Nat. Phys. 4, 2459-7 (2017).

93. Sandilands, L. J., Tian, Y., Plumb, K. W., Kim, Y.-J. \& Burch, K. S. Scattering continuum and possible fractionalized excitations in $\alpha-\mathrm{RuCl}_{3}$. Phys. Rev. Lett. 114, 147201 (2015).

94. Chun, S. H. et al. Direct evidence for dominant bond-directional interactions in a honeycomb lattice iridate $\mathrm{Na}_{2} \mathrm{IrO}_{3}$. Nat. Phys. 11, 462-466 (2015).

95. Gretarsson, $\mathrm{H}$. et al. Magnetic excitation spectrum of $\mathrm{Na}_{2} \mathrm{IrO}_{3}$ probed with resonant inelastic X-ray scattering. Phys. Rev. B 87, 220407 (2013).

96. Nasu, J., Knolle, J., Kovrizhin, D. L., Motome, Y. \& Moessner, R. Fermionic response from fractionalization in an insulating two-dimensional magnet. Nat. Phys. 12, 912-915 (2016).

97. Giamarchi, T. Quantum physics in one dimension. International Series of Monographs on Physics (Clarendon Press, Oxford, 2004).

98. Schlappa, J. et al. Spin-orbital separation in the quasi-one-dimensional Mott insulator $\mathrm{Sr}_{2} \mathrm{CuO}_{3}$. Nature 485, 82-85 (2012).

99. Bisogni, V. et al. Orbital control of effective dimensionality: from spin-orbital fractionalization to confinement in the anisotropic ladder system $\mathrm{CaCu}_{2} \mathrm{O}_{3}$. Phys. Rev. Lett. 114, 096402 (2015).

100. Chen, C.-C., van Veenendaal, M., Devereaux, T. P. \& Wohlfeld, K. Fractionalization, entanglement, and separation: understanding the collective excitations in a spin-orbital chain. Phys. Rev. B 91, 165102 (2015).

101. Yang, C. N. $\eta$ pairing and off-diagonal long-range order in a Hubbard model. Phys. Rev. Lett. 63, 2144-2147 (1989).

102. Zhang, S. Pseudospin symmetry and new collective modes of the Hubbard model. Phys. Rev. Lett. 65, 120-122 (1990).

103. Werner, P., Strand, H. U., Hoshino, S., Murakami, Y. \& Eckstein, M. Enhanced pairing susceptibility in a photodoped two-orbital Hubbard model. Phys. Rev. B 97, 165119 (2018).

104. Kitamura, S. \& Aoki, H. $\eta$-pairing superfluid in periodically-driven fermionic Hubbard model with strong attraction. Phys. Rev. B 94, 174503 (2016).

105. Kaneko, T., Shirakawa, T., Sorella, S. \& Yunoki, S. Photoinduced $\eta$ Pairing in the Hubbard model. Phys. Rev. Lett. 122, 077002 (2019).

106. Cook, M. W. \& Clark, S. R. Controllable finite-momenta dynamical quasicondensation in the periodically driven one-dimensional Fermi-Hubbard model. Phys. Rev. A 101, 033604 (2020).
107. Fujiuchi, R., Kaneko, T., Sugimoto, K., Yunoki, S. \& Ohta, Y. Superconductivity and charge density wave under a time-dependent periodic field in the one-dimensional attractive Hubbard model. Phys. Rev. B 101, $235122(2020)$

108. Peronaci, F., Parcollet, O. \& Schiró, M. Enhancement of local pairing correlations in periodically driven Mott insulators. Phys. Rev. B 101, 161101 (2020).

109. Sentef, M. A., Tokuno, A. Georges, A. \& Kollath, C. Theory of LaserControlled Competing Superconducting and Charge Orders. Phys. Rev. Lett. 118, 087002 (2017).

110. Tindall, J. et al. Dynamical Order and Superconductivity in a Frustrated Many-Body System. Phys. Rev. Lett. 125, 137001 (2020).

111. Suzuki, H. et al. Probing the energy gap of high-temperature cuprate superconductors by resonant inelastic x-ray scattering. npj Quantum Mater. 3, 65 (2018).

112. Ma, Y. \& Blume, M. Interference of fluorescence $\mathrm{x}$ rays and coherent excitation of core levels. Rev. Sci. Instrum. 66, 1543-1545 (1995).

113. Revelli, A. et al. Resonant inelastic x-ray incarnation of Young's doubleslit experiment. Sci. Adv. 5, p.eaav4020 (2019). Experimental observation of intermediate state interference in the RIXS spectrum of a dimerized oxide.

114. Abbamonte, $\mathrm{P}$. et al. Crystallization of charge holes in the spin ladder of $\mathrm{Sr}_{14} \mathrm{Cu}_{24} \mathrm{O}_{41}$. Nature 431, 1078-1081 (2004).

115. Zhang, J. et al. Stacked charge stripes in the quasi-2D trilayer nickelate $\mathrm{La}_{4} \mathrm{Ni}_{3} \mathrm{O}_{8}$. Proc. Natl Acad. Sci. USA 113, 8945-8950 (2016).

116. Zhang, J. et al. Large orbital polarization in a metallic square-planar nickelate. Nat. Phys. 13, 864-869 (2017)

117. Haldane, F. D. M. Model for a quantum Hall effect without Landau levels: condensed-matter realization of the "parity anomaly". Phys. Rev. Lett. 61, 2015 (1988).

118. Claassen, M., Jia, C., Moritz, B. \& Devereaux, T. P. All-optical materials design of chiral edge modes in transition-metal dichalcogenides. Nat. Commun. 7, 1-8 (2016).

119. Hübener, H., Sentef, M. A., De Giovannini, U., Kemper, A. F. \& Rubio, A. Creating stable Floquet-Weyl semimetals by laser-driving of 3D Dirac materials. Nat. Commun. 8, 13940 (2017).

120. Kitagawa, T., Oka, T., Brataas, A., Fu, L. \& Demler, E. Transport properties of nonequilibrium systems under the application of light: Photoinduced quantum Hall insulators without Landau levels. Phys. Rev. B 84, 235108 (2011).

121. Gu, Z., Fertig, H., Arovas, D. P. \& Auerbach, A. Floquet spectrum and transport through an irradiated graphene ribbon. Phys. Rev. Lett. 107, 216601 (2011).

122. Usaj, G., Perez-Piskunow, P. M., Torres, L. F. \& Balseiro, C. A. Irradiated graphene as a tunable Floquet topological insulator. Phys. Rev. B 90, 115423 (2014).

123. Torres, L. F., Perez-Piskunow, P. M., Balseiro, C. A. \& Usaj, G. Multiterminal conductance of a Floquet topological insulator. Phys. Rev. Lett. 113, 266801 (2014).

124. Dehghani, H., Oka, T. \& Mitra, A. Out-of-equilibrium electrons and the Hall conductance of a Floquet topological insulator. Phys. Rev. B 91, 155422 (2015).

125. Zhang, J. et al. Pressure-induced superconductivity in topological parent compound $\mathrm{Bi}_{2} \mathrm{Te}_{3}$. Proc. Natl Acad. Sci. 108, 24-28 (2011).

126. Kirshenbaum, K. et al. Pressure-induced unconventional superconducting phase in the topological insulator $\mathrm{Bi}_{2} \mathrm{Se}_{3}$. Phys. Rev. Lett111, 087001 (2013)

127. Zhou, Y. et al. Pressure-induced superconductivity in a three-dimensional topological material ZrTe 5 . Proc. Natl Acad. Sci. 113, 2904-2909 (2016).

128. He, L. et al. Pressure-induced superconductivity in the three-dimensional topological Dirac semimetal $\mathrm{Cd}_{3} \mathrm{As}_{2}$. npj Quantum Mater. 1, 16014 (2016).

\section{Acknowledgements}

We acknowledge E. Baldini, M. Buzzi, R. Comin, G. Coslovich, M.P.M. Dean, J. Freericks, A.A. Husain, D. Nicoletti, A.H. Reid, and K. Wohlfeld for valuable discussions. We also thank M.P.M. Dean for providing the original data from ref. ${ }^{61}$. The experiments reported in Fig. 2 were supported by the US Department of Energy, Office of Basic Energy Sciences grant no. DE-FG02-06ER46285. Use of the LCLS, SLAC National Accelerator Laboratory, is supported by the US Department of Energy, Office of Science, Office of Basic Energy Sciences under Contract No. DE-AC02-76SF00515. The calculation reported in Fig. 4 used resources of the National Energy Research Scientific Computing Center (NERSC), a US Department of Energy Office of Science User Facility operated under Contract No. DE-AC02-05CH11231.

\section{Author contributions}

Both authors, M.M. and Y.W., wrote the manuscript. 


\section{Competing interests}

The authors declare no competing interests.

\section{Additional information}

Supplementary information is available for this paper at https://doi.org/10.1038/s42005020-00447-6.

Correspondence and requests for materials should be addressed to M.M. or Y.W.

Reprints and permission information is available at http://www.nature.com/reprints

Publisher's note Springer Nature remains neutral with regard to jurisdictional claims in published maps and institutional affiliations. (c) (i) Open Access This article is licensed under a Creative Commons Attribution 4.0 International License, which permits use, sharing, adaptation, distribution and reproduction in any medium or format, as long as you give appropriate credit to the original author(s) and the source, provide a link to the Creative Commons license, and indicate if changes were made. The images or other third party material in this article are included in the article's Creative Commons license, unless indicated otherwise in a credit line to the material. If material is not included in the article's Creative Commons license and your intended use is not permitted by statutory regulation or exceeds the permitted use, you will need to obtain permission directly from the copyright holder. To view a copy of this license, visit http://creativecommons.org/ licenses/by/4.0/.

(C) The Author(s) 2020 